\title{
The anatomical basis of somaesthetic temporal discrimination in humans
}

\author{
F Lacruz, J Artieda, M A Pastor, J A Obeso
}

\begin{abstract}
Somaesthetic temporal discrimination (STD) is the ability to perceive as separate two successive somaesthetic stimuli applied to the same or different parts of the body. Paired electrical stimuli were applied to the index finger, using different time-intervals, to study the STD threshold (STDT) in 84 normal subjects and 51 patients with focal cerebral lesions. Abnormal STDT values were found on the affected side of patients with a lesion of the primary somatosensory cortex, and internal capsulethalamus. Lesions which did not produce sensory impairment but caused abnormal STDT were located in the posterior parietal cortex, the head of the caudate nucleus, the putamen, the medial thalamus and the lenticular nucleus. Frontal, temporal and occipital cortex lesions did not produce any abnormality in the STDT, but one patient with a bilateral lesion of the supplementary motor area (SMA) had abnormal STDT. These results indicate that normal perception of two somaesthetic stimuli as separate in time depends not only upon the integrity of the somatosensory pathway and primary somaesthetic cortex, but also of the posterior parietal cortex, SMA and subcortical structures such as the striatum and thalamus.
\end{abstract}

Somaesthetic temporal discrimination (STD) is the ability to perceive as separate two successive somaesthetic stimuli applied to the same or different cutaneous loci. Most studies of cutaneous sensation in humans have failed to consider in isolation the temporal component. However, somatosensory modalities such as vibratory sense, kinaesthesia, stereognosis and graphaesthesia require adequate temporal processing of afferent stimuli. ${ }^{1}$

Several techniques have been applied to assess visual and auditory temporal resolution in neurological patients. ${ }^{2-4}$ For instance, the critical flicker fusion frequency and double flash threshold tests are abnormal in patients with multiple sclerosis. ${ }^{56} \mathrm{Click}$ fusion and click counting tests have been used successfully to evaluate patients with cortical deafness. ${ }^{7}$ Conversely, STD has rarely been studied in patients with cerebral lesions. ${ }^{8}$ Normal STD should theoretically depend upon the functional integrity of peripheral and central somaesthetic pathways and the primary somatosensory cortex (PSC). A "time-organising system" which lies in the inferior parietal lobe and the bank of the superior temporal sulcus has also been implicated in the anatomical basis of STD. ${ }^{9}$

We have applied a simple test, using electrical stimulation of the index finger, to assess STD in a large number of untrained normal subjects of all ages and in patients with focal cerebral lesions.

\section{Subjects and method}

Fifty one neurologically stable patients with focal cerebral lesions demonstrated by CT and MRI brain scan, with no evidence of any other disturbance of the peripheral or CNS, were studied and compared with 84 normal untrained subjects. The mean (SD) age was $54 \cdot 2$ (18) (range 24-87) for patients and 44.75 (19.7) (range 12-83) for controls. All subjects were in good general condition, with a normal level of consciousness and had no language difficulty at the time of the study. Patients with a gnostic defect, right-left confusion or dyscalculia were not included in the study.

Patients were divided according to the distribution of the lesions demonstrated by CT brain scan and the presence or absence of a sensory defect in the hands. The latter was defined as normal when patients had no error in any somaesthetic test applied to the limb; mild defect when minimal errors were detected in fine sensory tasks, that is, recognising different fabrics; severe defect when clear exteroceptive and propioceptive abnormalities were present but patients could still feel electrical stimulation with high stimulus intensity. The primary complex of the SEP was defined as normal when the three typical waves $(\mathrm{N}-20 / \mathrm{P}-25 / \mathrm{N}-$ 33) were recorded on parietal electrodes with normal latency and amplitude; mild abnormality when the $\mathrm{N}-20$ or $\mathrm{P}-25$ waves were delayed by no longer than $5 \mathrm{~ms}$ but had normal amplitude, and severe abnormality when no SEP potentials were recorded or were barely distinguishable. To be considered without sensory defect patients had to have normal sensation clinically and show no abnormality of the SEPs. The stimulus consisted of a rectangular electrical pulse of $0.2 \mathrm{~ms}$ with an intensity of three times the sensory threshold generated by a Grass S-88 stimulator and was applied through ring electrodes attached to the index finger. In the same session, median nerve sensory potentials and cortical SEPs (from the scalp) were recorded using the standard technique. Somatosensory evoked potentials were 


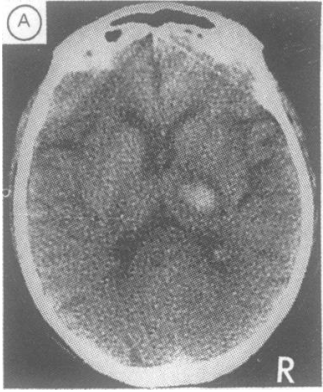

Figure 1 A) CT brain scan (without contrast) from a patient with hemisensory loss on the left side secondary to a postero-lateral right thalamic haematoma. The $R$ indicates position of the right hemisphere. $B$ ) $S E P$ s were normal in the right hand (left 4 channels) and abolished after left hand stimulation (right 4 channels). STDT values were $126 \pm 25 \mathrm{~ms}$ in the left hand and $36.2 \pm 6.3 \mathrm{~ms}$ in the right.
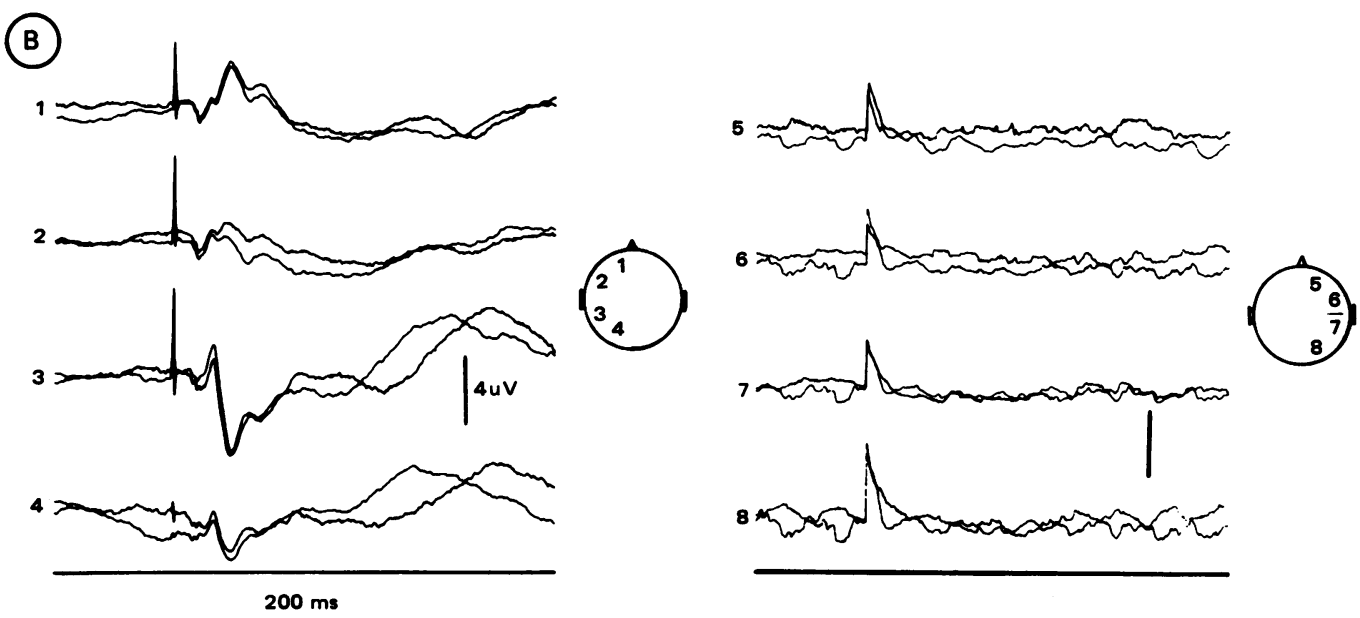

repeated twice for each patient after averaging a minimum of 128 responses. Each hand was independently studied in both controls and patients. In a few patients with a severe somaesthetic defect, increasing the stimulus intensity (up to six times the sensory threshold in the normal hand) was not sufficient to induce a sensation similar to the one evoked by stimulating the unaffected side. In such instances the stimulus intensity was adjusted to produce a sensation which the subject could reliably identify and which generated a sensory nerve potential with identical amplitude and latency to the one recorded in the normal hand.

With the above criteria, the three following groups were identified:

1) Patients with a capsulo-thalamic (12) or PSC (8) lesion which caused sensory loss. The lesion was in the right hemisphere in 13 patients and in the left in seven. The aetiology was infarction in 12, haemorrhage in five, tumour in two and post-traumatic scar in one;

2) Cortical without sensory defect (17). The topography of the lesions in these patients was anterior frontal in six (one in the right and five in the left hemisphere), temporal in four (one in the right and three in the left hemisphere) and occipital in four (two in the right and two in the left hemisphere). The aetiology of the lesion was infarction in eight, haemorrhage in three and tumour (glioma) in three. Two patients had

infarction of the posterior parietal (area 5-7) and one patient had a bilateral lesion (postsurgery) of the supplementary motor area (SMA).

3) Subcortical without sensory defect. This group includes 14 patients with no evidence of damage to the somesthetic pathways. Lesions were sited in the head of the caudate nucleus and the anterior limb of the internal capsule (5), the putumen (3), the thalamus $(4,3$ medially and 1 anterolaterally placed) and the right lenticular nucleus in one patient and bilaterally in two (5). The aetiology was infarction in 12 patients (thromboembolic in two and atherosclerotic lacunar in 10) and anoxia and cyanide poisoning in the two patients with bilateral lenticular lesions. None of the patients included in this group had large lesions causing distortion or impinging upon the ventricles and brainstem. Patients were studied in a chronic stage when oedema had probably disappeared and was not detected by CT scan.

\section{Method}

The tests were carried out in a quiet room at a temperature of $20-22^{\circ} \mathrm{C}$ with the subjects comfortably lying on a couch. The total duration of each test varied between one to three hours including resting periods.

The temporal discrimination threshold was

Table Distribution of lesions and effect on somaesthetic temporal discrimination threshold (STDT)

\begin{tabular}{|c|c|c|c|}
\hline \multirow[b]{2}{*}{ Topography of the lesion } & \multirow[b]{2}{*}{$\begin{array}{l}\text { Number of } \\
\text { patients }\end{array}$} & \multicolumn{2}{|l|}{ STDT Values (ms) } \\
\hline & & $\begin{array}{l}\text { Normal side } \\
\text { mean, } S D \text { (range) }\end{array}$ & $\begin{array}{l}\text { Side with lesion } \\
\text { mean, } S D \text { (range) }\end{array}$ \\
\hline \multicolumn{4}{|l|}{ A With sensory defect and abnormal STDT } \\
\hline $\begin{array}{l}\text { Cortex S-I and thalamo-cortical radiations } \\
\text { Capsulo-Thalamic }\end{array}$ & $\begin{array}{r}8(\mathbf{R 7}, \mathrm{L} 1)^{1} \\
12(\mathrm{R} 6, \mathrm{~L} 6)\end{array}$ & $\begin{array}{l}29 \cdot 2,11 \cdot 3(15-4 \cdot 7) \\
31 \cdot 8,5 \cdot 3(20-41 \cdot 2)\end{array}$ & $\begin{array}{l}627 \cdot 3,199 \cdot 2^{\star}(318 \cdot 75-900) \\
113,22 \cdot 4^{\star}(80-150)\end{array}$ \\
\hline \multicolumn{4}{|c|}{ B Without sensory defect and with normal STDT } \\
\hline Prefrontal, temporal, occipital & $14(\mathrm{R} 4, \mathrm{~L} 10)$ & $28 \cdot 8,7 \cdot 2(18 \cdot 7-38 \cdot 7)$ & $29 \cdot 6,8 \cdot 7(15-43)$ \\
\hline $\begin{array}{l}\text { C Without sensory defect and with abnorma } \\
\text { Posterior parietal } \\
\text { SMA } \\
\text { Anterior Capsule-Head of caudate nucleus } \\
\text { Putamen } \\
\text { Lenticular nucleus } \\
\text { Thalamus ( } 3 \text { medial, } 1 \text {, anterolateral) }\end{array}$ & $\begin{array}{l}2 \text { (R1, L1) } \\
1 \text { (bilateral) } \\
5(\mathbf{R} 1, \mathrm{~L} 4) \\
3(\mathbf{R} 2, \mathrm{~L} 1) \\
1 \mathrm{R} 2 \text { bilateral } \\
4 \text { (R2, L2) }\end{array}$ & $\begin{array}{l}25,27 \\
\overline{27} \cdot 5,9 \cdot 75(21 \cdot 25-41 \cdot 25) \\
29 \cdot 5,8 \cdot 9(20-37 \cdot 5) \\
32 \cdot 3 \\
30 \cdot 9,5 \cdot 1(26 \cdot 25-38 \cdot 75)\end{array}$ & $\begin{array}{l}127 \cdot 3 / 130 \cdot 9 \\
85 \cdot 1(L) / 105(R) \\
95 \cdot 2,11 \cdot 1 \star(85-108 \cdot 75) \\
80,8 \cdot 2(72 \cdot 5-78 \cdot 7)^{\star} \\
88 \cdot 7,7 \cdot 6(76 \cdot 2-88 \cdot 7) \\
60,4 \cdot 3 \star(56 \cdot 2-66)\end{array}$ \\
\hline
\end{tabular}

${ }^{1}$ Lesion in the right $(R)$ or left $(L)$ hemisphere. 


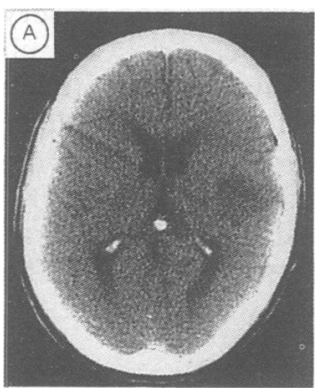

Figure 2 A) CT brain scan (without contrast), above the level of the third ventricle showing a small rolandic infarction. B) Cortical SEPs after right index finger stimulation were normal in the left hemisphere (left 4 channels) and abolished in the right hemisphere (right 4 channels) after left index finger stimulation. STDT values were $820 \pm 115 \mathrm{~ms}$ in the left hand and $33 \pm 11 \mathrm{~ms}$ in the right hand.
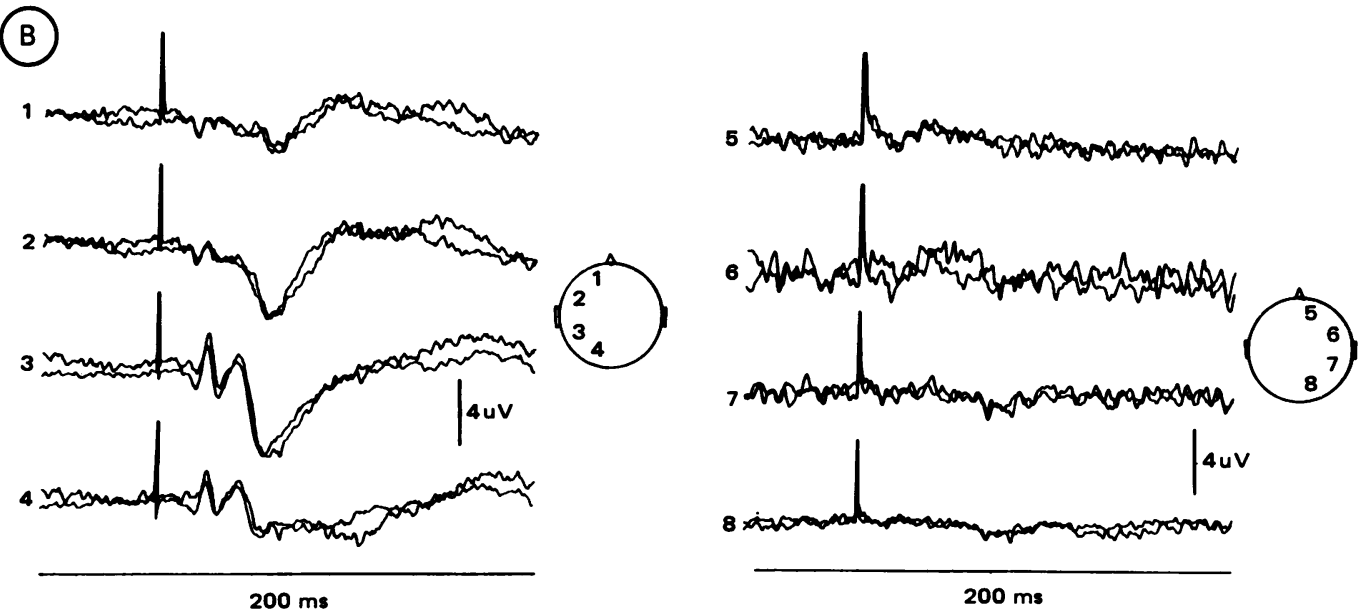

evaluated by applying the "method of limits". ${ }^{10}$ The minimal time-interval required for a pairstimuli to be felt as separated in time was assessed by increasing the time-difference between the stimuli, starting with a $5 \mathrm{~ms}$ interval, until the subject recognised the double stimulation (ascending temporal discrimination threshold = ATDT). Subsequently, starting with an interstimuli difference that allowed clear separation of both stimuli, the intervals were reduced until the subject could only recognise one stimulus (descending temporal discrimination threshold = DTDT). Both series were repeated on six occasions using different time-intervals intermixed randomly and also introducing pair-stimuli with a zero time-interval to minimise the risk of a learning and prediction effect upon the answers. This procedure also allowed us to check that due attention was maintained by the subject under examination. The arithmetic mean of both threshold values (ascending and descending TDT) was used as the final figure for statistical evaluation. Between the minimal and maximal threshold values there existed a period of "discriminative hesitation", at which timeintervals many subjects had difficulty in deciding whether the stimulation consisted of a single or double shock. Such sensations were described as "one but longer or stronger stimulus", or "two but almost continuous stimuli". In such instances the experimenter always proceeded to take as the STDT value a shorter or longer time-interval which gave rise to a clearly unitary or dual sensation. Before starting the study, the subjects received a clear explanation of the nature and purpose of the test and practical examples of double and single stimulation were given to ensure that they understood the method. Special emphasis was given to the absence of a time constraint when giving the answer, and that the test could be repeated as many times as necessary before producing a definitive answer. Subjects (both control and patients) showing signs of distraction, difficulty in reliably carrying out the test or, who did not tolerate electrical stimulation to the index finger were not included in this investigation.
In five normal subjects the study was repeated on five different days to assess the reproducibility of the test. No significant intraindividual nor inter-individual variation (twoways ANOVA with replication) for the STDT values were found in these subjects upon repeated examination on different days. Results are reported as the mean standard deviation and range. ANOVA and $t$ test were used for statistical analysis.

\section{Results \\ A) General findings}

The mean (SD) STDT value in the control group was $30.68(8.87) \mathrm{ms}$ (range 15-55.65) in the right and $30.77(8.78) \mathrm{ms}$ (range 15-58.75) in the left hand $(t=0.97 p>0.05)$. The mean (SD) STDT value for the patient group was $29.65(7.36) \mathrm{ms}(15-43)$ on the normal side (ipsilateral to the lesion) and $172 \cdot 75(225.9) \mathrm{ms}$ (range 15-900) in the affected hand (contralateral to the lesion; $t=4.20 ; p<0.001)$. Thirty seven patients had a mean (SD) STDT values $(233.5(246 \cdot 1) \mathrm{ms}$, range 61-900) falling at least three standard deviation beyond the mean normal value.

In patients with a left cortical lesion $(n=13)$ mean (SD) STDT on the contralateral hand was 103.6 (167) ms (range 15-575) and in patients with a right cortical lesion $(n=13)$ mean (SD) STDT (contralateral hand) was 339.67 (339.95) $\mathrm{ms}$, (range 16.25-900; $t=$ $2.02 ; p>0.05)$. There was also no significant difference in the STDT values $(t=1.446$; $p>0.05$ ) obtained in the affected hand between patients with subcortical lesions in the right $(\mathrm{n}=14)$ [STDT $=108.9(23.4) \mathrm{ms}$, range 56.25-126] and left hemisphere $(n=15)$ [STDT $=94.7(20.5) \mathrm{ms}$, range 58.75-120].

No patient had an abnormal STDT on the side ipsilateral to the lesion.

\section{B) Anatomical Correlations}

Patients with a severe sensory defect secondary to a lesion of the somaesthetic pathway and PSC showed the greatest defect in STDT (table 1-A). The effect of somaesthetic cortex lesion (S-I) was significantly greater [STDT 


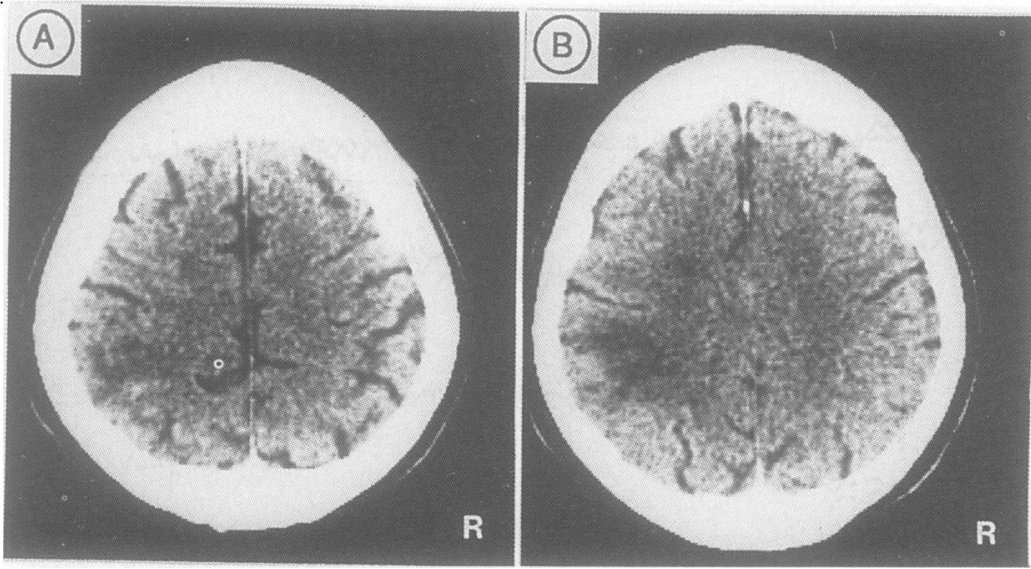

(C)
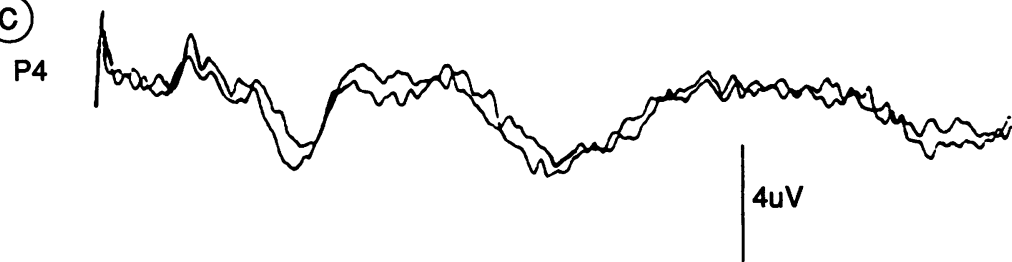

P3

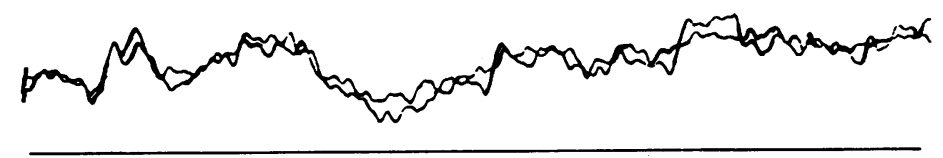

200ms

Figure $3 C T$ brain scan ( $A$ and $B$ ) above the lateral ventricle shows an area of hypodensity in the left posterior parietal lobe. C. Somatosensory evoked potentials, recorded over both hemispheres (P3 and P4), showed a normal primary complex bilaterally and a minor asymmetry of latter waves due to flattening of the P5O-N90 wave on the affected side (P3). This patient had an STDT value of $208.75 \pm 13.5 \mathrm{~ms}$ in the right hand and of $25 \pm 7.8 \mathrm{~ms}$ in the left hand.

$627 \cdot 3(199 \cdot 2)$ versus $113(22 \cdot 4), \mathrm{p}<0 \cdot 001]$ than capsulo-thalamic lesion, even when the degree of sensory loss was similar (figs 1 and 2). Lesions located in the anterior pole of the frontal lobe, temporal and occipital lobe caused no abnormality in the STDT (table 1-B).

In 17 patients (table 1-C) in whom no sensory loss was appreciated on clinical and electrophysiological examination (figs 3 and 4), the STDT was abnormal, although the actual values (table $1-\mathrm{C}$ ) were not as high as those seen in patients with PSC lesion. The sensory threshold for detection of the electrical stimuli was absolutely normal in this subgroup (table -C) compared with either their normal side or control subjects. Interestingly, lesions of the posterior parietal cortex produced STDT values (mean $129.2 \mathrm{~ms}$ ) slightly higher than subcortical capsulo-thalamic lesions of the somethetic pathway [mean (SD) $113(22.4)$ ms].

\section{Discussion}

Normal values for STDT in the upper limb varied from 10 to $40 \mathrm{~ms}$ in most studies. ${ }^{811-13}$ Our results in normal subjects confirm previous findings. The technique applied in the study is easy to perform and the values obtained are fairly constant for the normal population. The time course of the peripheral nerve recovery curve does not explain STDT values because the amplitude of the sensory nerve action potential is $100 \%$ recovered for time-intervals of 5-10 ms. ${ }^{1415}$ Accordingly, a subject's inability to distinguish two stimuli with intervals above $10 \mathrm{~ms}$ must depend upon central sensory mechanisms.

In the only previous study of the STDT in patients with neurological disorders, Green et $a l^{8}$ found that peripheral nerve and posterior column lesions caused no abnormality in the STDT. Our results also indicate that the somatosensory cortex, when spared, can partially compensate for the defective arrival of cutaneous input. On the other hand, lesions of the PSC caused the greatest abnormality in the STDT, in keeping with the paramount role of this area in somaesthetic function. The great effect of posterior parietal cortex lesions on the STDT strongly suggests that this region is involved in the temporal perception and resolution of paired stimuli. This is not surprising since unicellular recording of neuronal responses in the posterior parietal cortex indicates that areas 5 and 7 are highly activated by joint and skin stimulation, particularly during discriminative tasks. ${ }^{16}$ General
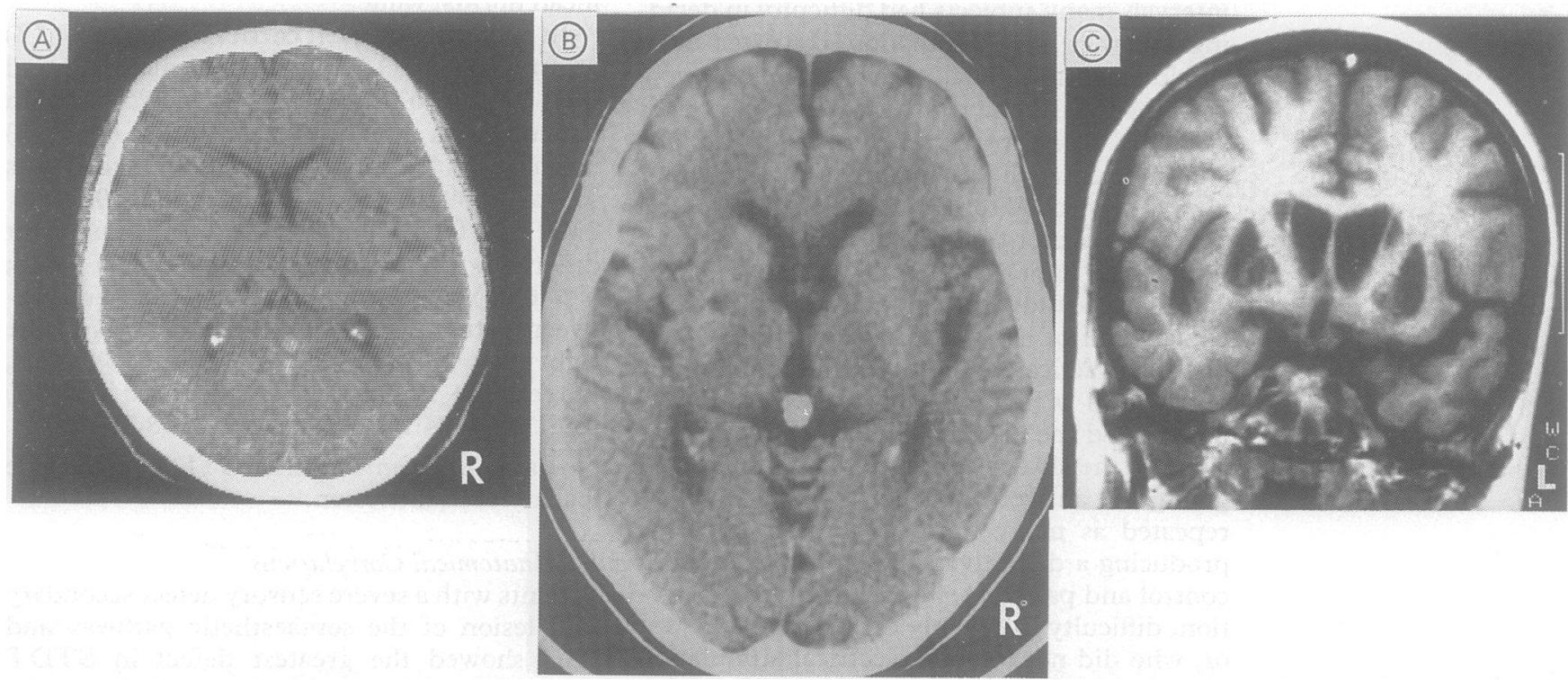

Figure $4 C T$ brain scan ( $A$ and $B$ ) at the level of the third ventricle from patients with focal lesions sparing the somatosensory pathway but abnormal STDT (see table). A. Left medial thalamic infarction. B. Left putaminal infarction. C. Bilateral lenticular and caudate nuclei hypoxic infarction. 
requirements for normal temporal discrimination are that the subject is alert and capable of focusing attention on the stimulated limb, while ignoring other non-relevant stimuli, which may occur during the test. Sensory tasks requiring the subjects to direct their attention to a finger to detect threshold stimuli are associated with increased cerebral blood flow in the contralateral somatosensory cortex, but also in the posterior parietal region and prefrontal cortex. ${ }^{17}$ Growing evidence has accumulated on the specialised role of the right hemisphere in attention and intention related to external space. ${ }^{1819}$ The very abnormal STDT values in patients with parietal lesion could have been due not only to the critical role and areas 5 and 7 in somaesthetic processing, but because the lesion was in the right hemisphere in eight of the 11 patients. Conversely, no difference in STDT values for patients with right and left subcortical lesions was seen.

The high incidence of abnormal STDT in patients with basal ganglia and mediolateral thalamic lesions was totally unexpected, since these structures are primarily involved in motor control. ${ }^{20} \mathrm{We}$ do not believe that a nonspecific effect of the lesions included here could explain these results. In most patients the lesion consisted of infarction restricted to the putamen, caudate and thalamic nuclei and the study was conducted when the acute effect of the lesion had disappeared. Furthermore, neuroimaging in these patients show neither displacement nor collapse of the third ventricle which could compromise the somatosensory pathways. The main cortical output from the basal ganglia goes via the lateral thalamus to the SMA, which in turn projects heavily to the superior parietal lobe. ${ }^{21}$ Neuronal firing in prefrontal and premotor areas is enhanced during new cognitive tasks. ${ }^{22}$ It is possible that basal ganglia and thalamic lesions could negatively influence the neuronal activity underlying the normal STDT by way of their projections to the SMA. The finding of an abnormal STDT in the only patient we were able to study with a bilateral SMA lesion agrees with this explanation, which nevertheless must be considered preliminary and subject to further testing.

Recently, Ivry and $\mathrm{Keele}^{23}$ reported an abnormal capacity of patients with lesions of the cerebellum to discriminate small changes in the time interval between two consecutive auditory tones. In addition, cerebellar patients showed a deficit in producing repetitive finger movements in accordance with an externally given rhythm. From these observations Ivry and Keele ${ }^{24}$ proposed that the cerebellum plays an essential timing function, not only related to motor control but also to perceptive and cognitive mechanisms. Our experience certainly indicates that integrity of other brain structures is equally essential to guarantee normal time perception. Arguably, the brain areas whose integrity we have found necessary for normal STD are specific to the somatosensory modality, while the cerebellum could play a more general rcle in time perception. In Parkinson's disease, however, we have found a marked alteration of the TDT for somaesthetic visual and auditory stimuli without any primary sensory defect. ${ }^{25}$ Thus we believe that the basal ganglia also has a general role in temporal discrimination across modalities.

We are grateful to Miss M Mar López and Carol Elsden for preparing and editing this paper. Dr J L Zubieta (Neuroradiology Unit) reviewed and commented on the neuroimaging studies.

1 Mountcastle VB. Neuronal mechanisms in somesthesis. In Mountcastle VB, ed. Medical physiology, 14th ed. Louis: CV Mosby, 1980;348-90.

2 Goldman PS, Lodge A, Hammer LR, Semmes J, Mishkin $M$. Critical flicker frequency after unilateral temporal lobectomy in man. Neuropsychologia. 1968;5:355-63.

3 Lackner JR, Teuber HL. Alterations in auditory fusion threshold after cerebral injury in man. Neuropsychologia, 1973;11:409-15.

1 Ricklan M, Levita E, Misiak H. Cortical flicker frequency and integrative functions in Parkinsonism. $J$ Psychol and integrative

5 Galvin RJ, Regan D, Heron JR. Impaired temporal resolution of vision after acute retrobulbar neuritis. Brain 976;99:255-68.

6 Plant GT, Hess RF. Temporal frequency discrimination in optic neuritis and multiple sclerosis. Brain 1985;108: 647-76

7 Albert ML, Bear D. Time to understand. A case study of word deafness with reference to the role of time in auditory comprehension. Brain 1974;97:373-84.

8 Green JB, Reese Ch.L, Pegues JJ, Elliot FA. Ability to distinguish two cutaneous stimuli separated by a brief time interval. Neurology 1961;11:1006-10.

9 Tanaka J, Yamadori A, Mori E. Pure word deafness following bilateral lesions. Brain 1987;110:381-403.

10 Engen T. Psychophysics. In: Kling JW, Riggs LA, eds. Woodworth and Scholosberg's experimental psychology, rd ed. London: Methuen, 1972:14-20.

11 Rosner BS. Neural factors limiting cutaneous spatiotemporal discriminations. In: Rosenblith WA, ed. Sensory communications. New York: MIT Press, 1961:725-37.

12 Axelrod S, Thompson LW, Cohen LD. Effects of senescence on the temporal resolution of somesthetic stimuli presented to one hand or both. J Geront $1968 ; 23: 191-5$.

13 Fucci A, Petrosino L. Cutaneous temporal resolution values for sophisticated and naive subjects. Perceptual and Motor Skills 1984;58:831-4.

14 Shagass CH, Schwartz M. Recovery function of somatosensory peripheral nerve and cerebral evoked responses in sensory peripheral nerve and cerebral evoked responses

$15 \mathrm{Ng} \mathrm{A}$, Burke D, Al-Shehab A. Hyperexcitability of cutaneous afferents during the supernormal period. Brain 1987;110:1015-31.

16 Hyvarinen J. The parietal cortex of monkey and man. Berlin: Springer Verlag, 1982:76-99.

17 Roland PE. Somatotopical tuning of postcentral gyrus during focal attention in man. A regional cerebral blood flow study. $J$ Neurophysiol 1981;46:744-54.

18 Heilman KM, Van Den Abell T. Right hemisphere dominance for attention: The mechanism underlying hemisphere asymmetries of inattention (neglect). Neurology 1980;30:327-30.

19 Weintraub S, Mesulam MM. Right cerebral dominance in spatial attention. Further evidence based on ipsilateral neglect. Arch Neurol 1987;44:621-5.

20 Alexander GE, De Long MR, Strick PL. Parallel organisation of functionally segregated circuits linking basal ganglia and cortex. Ann Rev Neurosc 1986;9:357-81.

21 Jurgens U. The efferent and afferent connexions of the urgens $U$. The efferent and afferent connexions of the
supplementary motor area. Brain Res 1984;300:63-81.

22 Fuster JM, Baner RH, Jervey JP. Cellular discharge in the dorsolateral prefrontal cortex of the monkey in cognitive tasks. Exp Neurol 1982;77:679-94.

23 Ivry RB, Keele SW, Diener HC. Dissociation of the latera and medial cerebellum in movement timing and movement execution. Exp Brain Res 1988;73:167-80.

24 Ivry RB, Keele SW. Timing functions of the cerebellum $J$ Cognitive Neuroscience 1989;1(2):136-51

25 Artieda J, Pastor MA, Lacruz F, Obeso JA. Tempora discrimination is abnormal in Parkinson's disease. Brain (In Press). 